\title{
Ueber neue Derivate des Digitogenins.
}

\author{
Von H. Kiliani und M. Bazlen.
}

(Eingegangen am 29. VI. 1894.

In der letzten Abhandlung uber obigen Gegenstand ${ }^{1}$ ) wurde die Vermutang ausgesprochen, dafs „die Digitsäure und mit ihr das Digitogenin, sowie dessen sonstige Derivate in naber Beziehung zu den Terpenen stehen." Auch die Möglichkeit, hierfür einen einfachen experimentellen Beweis zu erbringen, schien bereits gegeben, insofern es gelungen war, unter den Oxydationsprodukten der Digitsäure-Mutterlaugen eine zweibasische Säure $\mathrm{C}_{9} \mathrm{H}_{14} \mathrm{O}_{4}$ aufzufinden, welche möglicherweise identisch war mit der Isopyrocamphersäure von $\mathrm{Marsh}$ und $\mathrm{G}$ a r d n er. Diese Hoffnung wurde leider getäuscht, doch gelang es wenigstens durch anderweitige Versuche im Abbau der Digitsiiure, einen wesentlichen Fortschritt zu erzielen. Hierüber, sowie über verschiedene sonstige Erfahrungen, welche inzwischen gesammelt wurden, soll im Folgenden berichtet werden.

Verbesserte Darstellung der Digitogensäure.

Das Ausgangsmaterial für diese Untersuchung, das Digitonin, ist ziemlich kostspielig und kann in grölserer Menge überhaupt nur schwer beschafft werden; überdies schrumpft dasselbe schon durch die Abspaltung der beiden Zuckermoleküle auf 30 Proz. (theoretisch 36,8 Proz.) $)^{2}$ ) seiner ursprünglichen Grölse zusammen. Nun erhält man weiter aus dem Digitogenin 60 Proz. Digitogensäure, und aus dieser endlich 30 Proz. Digitsäure, d. h. aus $100 \mathrm{~g}$ reinem krystallisiertem Digitonin können auch bei grölster Sorgfalt, und obwohl die Einzelausbeuten nicht geradezu schlecht sind, nur 100 . 0,3 . 0,6 . 0,3 $=5,4 \mathrm{~g}$ Digitsäure gewonnen werden, also eine recht kärgliche Menge. Jede Vermehrung der Ausbeute an Digitogensäure oder Digitsäure wäre also freudigst zu begrülsen. Wir haben deshalb in dieser Richtung eine Reihe von Versuchen angestellt, bedauerlicher Weise aber nur negative Resultate erhalten. Lediglich in Bezug auf Einfachheit und Billigkeit vermochten wir die Darstellung der Digitogensäure bedeutend vorteilhafter zu gestalten.

1) Dieses Archiv 1893. S. 448.

2) S. dieses Archiv Bd. 230. S. 261. 
Die Gewinnung dieser Säure nach der älteren Vorschrift ${ }^{3}$ ) bedingt die Anwendung von sehr viel Eisessig und in Folge davon beim Ausschütteln des Rohproduktes durch Aether einen überaus grolsen Verbrauch auch an letzterem, so dals das ganze Verfahren, wenn es sich um die Darstellung gröfserer Quantitäten handelt, zu einem recht lästigen und auch kostspieligen wird.

Der nächstliegende Gedanke, eine Vereinfachung dadurch eintreten zu lassen, dals man nach vollendeter Oxydation die Hauptmenge der Essigsäure durch Abdampfen entfernt und dann die Digitogensäure direkt auskrystallisieren läfst, oder eventuell durch Aether ausschüttelt, erwies sich als praktisch undurchführbar, weil beim Eindampfen der weitaus grölste Teil der Digitogensäure in chromhaltige schmierige Produkte verwandelt wird. Wohl aber kann man die Menge des Eisessigs von vornherein bedeutend reduzieren (bis auf 26 Proz. der l. c. angegebenen Gröfse) und dadurch die ganze Arbeit wesentlich erleichtern. Man benützt zweckmälsig folgendes Verfahren.

1 T. Digitogenin wird mit $10 \mathrm{~T}$. Eisessig übergossen und im Verlaufe von 3 Stunden allmählich unter fleifsigem Umschwenken mit einer Lösung von $0,7 \mathrm{~T}$. kryst. Chromsäure in $1,4 \mathrm{~T}$. Wasser versetzt, wobei sich das Digitogenin langsam auflöst. Nach weiterem mehrstiundigem Stehen fugt man noch $10 \mathrm{~T}$. Wasser hinzu und schüttelt viermal mit Aether. Im Uebrigen verfuhrt man wio früher angegeben, nur kann zum Umkrystallisieren des Rohproduktes auch 93 proz. Alkohol an Stelle des damals empfohlenen absoluten verwendet werden. Die Ausbeute beträgt ebenso wie sonst ca. 60 Proz.

$$
\text { Oxim der Digitogensäure. }
$$

Diese Verbindung, deren Existenz schon in der letzten Publikation angedeutet wurde, erhält man durch Auflösen von $1 \mathrm{Mol}$. Digitogensüure in $3 \mathrm{Mol}$. Natronhydrat ( $30 \mathrm{~g}$ in 1 Liter Wasser), $\mathrm{Zu}$ satz von etwas mehr als $1 \mathrm{Mol}$. salzsaurem Hydroxylamin, 12-24 stündiges Stehenlassen der Mischung, Zugabe von 93 prozentigem Alkohol (20 Proz. vom Gewichte der ganzen Lösung) und schliefsliches Ansäuern mittelst Salzsäure. Der flockig-körnige Niederschlag

3) Ber. d. d. chem. Ges. XXIV. 342. 
wird mit Wasser gewaschen, auf Thon getrocknet und in $5 \mathrm{~T}$. 93 prozentigen Alkohols durch schwaches Erwärmen gelöst; nun wird filtriert und zur Lösung das gleiche Gewicht Wasser gegeben, worauf rasch eine starke Krystallisation (derbe Nadeln oder Prismen) entsteht. Man saugt ab, wäscht mit möglichst wenig 50 prozentigem Alkohol und trocknet das Material an der Luft.

Das Produkt ist stickstoffhaltig, leicht löslich in Alkohol und in Aether, sehr wenig löslich in Wasser, und wenn trocken, von diesem schwer benetzbar. Es reagiert sauer, löst sich in Soda unter Aufbrausen und besitzt im Gegensatze zur Mehrzahl der übrigen Digitogeninderivate einen ziemlich scharfen Schmelzpunkt, nämlich $175^{\circ}$.

Leider gaben die 10 Elementaranalysen, welche ausgeführt wurden, weder unter sich noch mit der Theorie übereinstimmende Werte, weil die Substanz eine äufserst schwer verbrennliche Kohle hinterlälst, die wahrscheinlich stickstoffhaltig ist. Gefunden wurden nämlich im Mittel 4,2 Proz. Stickstoff (ber. für $\mathrm{C}_{14} \mathrm{H}_{23} \mathrm{O}_{4} \mathrm{~N}$ 5,2 Proz.) und der Kohlenstoff fiel um so höher aus, je länger die Substanz vorher auf $110^{\circ}$ erhitzt worden war, wobei sie aber sehr schwer konstantes Gewicht annahm und im Mittel einen Gewichtsverlust von 5,5 Proz. erlitt. Irgend eine Zersetzung war beim Erhitzen nicht eingetreten, denn das Oxim blieb rein weifs, behielt den ursprünglichen Schmelzpunkt und die gleichen Löslichkeitsverhältnisse. Auch ein Gemenge lag nicht vor, wie uns eine sorgfältig durchgeführte fraktionierte Krystallisation bewies. Erst die Untersuchung der Salze brachte Klarheit über diese Verhältnisse.

Magnesiumsalz. Löst man das Oxim in der äquivalenten Menge Kalilauge, verdünnt dann bis 1:150 und giebt endlich Magnesiumnitrat $(1: 10)$ hinzu, so scheiden sich erst nach längerem Stehen hubsche lange Nadeln des Magnesiumsalzes aus. In konzentrierterer Lösung (etwa 1:50) entsteht sofort ein dicker gallertartiger Niedersclag, der sich innerhalb 2 Tagen in weifse Krystallwärzchen umwandelt.

$0.2188 \mathrm{~g}$ bei $120^{0}$ getrocknetes Salz lieferten $0,0172 \mathrm{~g} \mathrm{MgO}$.
Ber. für $\left(\mathrm{C}_{14} \mathrm{H}_{20} \mathrm{O}_{3} \mathrm{~N}\right)_{2} \mathrm{Mg}$
Gefunden 4,70

B a r y u msa lz. Beim Vermischen der neutralen Kalisalzlösung (1:350) mit Chlorbaryum $(1: 10)$ entsteht ein schwacher amorpher 
Niederschlag. Man filtriert sofort in einen Kolben, überschichtet die Lösung mit ganz wenig Alkohol und lälst einige Stunden ruhig stehen. Es bilden sich lange, zu Büscheln vereinigte Nadeln, welche mit möglichst wenig Wasser zu waschen sind.

I. $0.271 \mathrm{~g}$ lufttrockenes Salz verloren bei $120^{0} 0,0394 \mathrm{~g} \mathrm{H}_{2} \mathrm{O}$.

II. $0,176 \mathrm{~g}$ wasserfreies Salz lieferten $0,0544 \mathrm{~g} \mathrm{Ba} \mathrm{CO}$.

III. $0,2398 \mathrm{~g}$ wasserfreies $\mathrm{Salz}$ lieferten $0,074 \mathrm{~g} \mathrm{Ba} \mathrm{CO}$.

Ber. fur $\left(\mathrm{C}_{14} \mathrm{H}_{20} \mathrm{O}_{3} \mathrm{~N}_{2} \mathrm{Ba}+6 \mathrm{H}_{2} \mathrm{O}\right.$ Gefunden

$\mathrm{H}_{2} \mathrm{O} \quad 14,49$

14,53

Ber. für $\left(\mathrm{C}_{14} \mathrm{H}_{22} \mathrm{O}_{4} \mathrm{~N}\right)_{2} \mathrm{Ba}$, für $\left(\mathrm{C}_{14} \mathrm{H}_{20} \mathrm{O}_{3} \mathrm{~N}\right)_{2} \mathrm{Ba}$

$\mathrm{Ba} \quad 20,35$

21.5

Gefunden

Die Zusammensetzung der Salze zoigt also, dafs das Oxim nicht die erwartete Formel $\mathrm{C}_{14} \mathrm{H}_{23} \mathrm{O}_{4} \mathrm{~N}$ besitzt; es entsteht vielmehr überraschender Weise nach der Gleichung

$$
\mathrm{C}_{14} \mathrm{H}_{22} \mathrm{O}_{4}+\mathrm{NH}_{2} \mathrm{OH}=\mathrm{C}_{14} \mathrm{H}_{21} \mathrm{O}_{3} \mathrm{~N}+2 \mathrm{H}_{2} \mathrm{O}
$$

d. h. unter Abspaltung eines zweiten Moleküls Wasser.

Das lufttrockene Oxim enthält ferner Krystallwasser, welches bei $110^{\circ}$ nicht vollständig entweicht, und deshalb, sowie wegen der schweren Verbrennlichkeit der entstehenden Kohle erhält man für Stickstoff und Kohlenstoff so schwer konstante und richtige Zahlen. Nur eine mittelst chromsaurem Blei ausgeführte Analyse des bei $110^{\circ}$ getrockneten Oxims ergab einen annähernd brauchbaren Wert, nämlich 66,13 Proz. $C$ statt der für $\mathrm{C}_{\mathbf{1 4}} \mathrm{H}_{\mathbf{2 1}} \mathrm{O}_{3} \mathrm{~N}$ berechneten 66,93 Proz.

Eine Diskussion der Frage, in welcher Weise bei der Bildung des Oxims das zweite Mol. Wasser austritt, ist erst nach weiterer Autklärung der Konstitution möglich.

Esterifizierung der Säuren aus Digitogenin.

Da bisher von keiner der hierher gehörigen Säuren Ester bokannt waren, erschien es von Interesse, deren Darstellung zu versuchen. Wir wählten dazu Digitsäure, Digitosäure und die später zu erwähnende Acetyl- $\beta$-Anhydrodigitsäure. Je 1 T. Säure wurde in 5 T. absoluten Alkohols gelöst und trockenes Salzsäuregas unter Eiskühlung bis zur Sättigung eingeleitet. Nach mehreren Stunden wurde in Wasser gegossen und der entstandene Niederschlag gereinigt. Aber nur aus der Digitosăure erbielten wir ein krystallisiertes Produkt. 
Der Digitosäureäthylester lälst sich leicht aus kochendem 93 proz. Alkohol umkrystallisieren. Er bildet derbe Nadeln oder Prismen, beginnt schon bei $120^{\circ}$ zu sintern, schmilzt aber vollständig erst gegen $160^{\circ}$.

$0,1488 \mathrm{~g}$ gaben $0,3880 \mathrm{~g} \mathrm{CO}_{2}$ und $0,1264 \mathrm{~g} \mathrm{H}_{2} \mathrm{O}$.

Berechnet für $\mathrm{C}_{15} \mathrm{H}_{24} \mathrm{O}_{3} \quad$ Gefunden

C 71,43 Proz.

71,44 Proz

H $9,52,9,44$,

Oxydation der Nebenprodukte von der Dar stellung der Digitsäure.

Die früher durch Oxydation der Digitsäure-Mutterlaugen gewonnene zweibasische Săure $\mathrm{C}_{9} \mathrm{H}_{14} \mathrm{O}_{4}$ zeigte nahezu die gleichen Eigenschaften, wie sie die Isopyrocamphensäure nach der $\mathrm{Be}$ schreibung von Marsh und Gardner ${ }^{1}$ ) besitzen sollte. Um nun den direkten Vergleich zu ermöglichen, war beabsichtigt, die zuletzt genannte Säure nach den Angaben der englischen Forscher darzustellen, was jedoch nicht gelang. Herr Dr. Sanda, welcher diesen Versuch ausführte, erhielt vielmehr bei der trockenen Destillation der Camphensäure nur e in e Säure, die Pyrocamphensäure. Dieselbe krystallisierte aus kochendem Wasser beim Erkalten sofort in prächtigen, zu Warzen vereinigten Prismen aus und gab kein saures Kalisalz von der Formel $\mathrm{C}_{9} \mathrm{H}_{13} \mathrm{O}_{4} \mathrm{~K}+\mathrm{C}_{9} \mathrm{H}_{14} \mathrm{O}_{4}$, war also völlig verschieden von unserer Säure. ${ }^{2}$ )

Dann sollte letztere Verbindung behufs genauerer Untersuchung in grörserer Menge gewonnen werden, was ebenfalls mirslang. Dies wurde

1) Journ, chem. soc. 59, 648.

2) Bei der Darstellung der Camphensüure ist es zweckmä ${ }^{3}$ ig, nach dem Verdampfen der überschüssigon Salpetersäure den verbleibenden Sirup mit dem gleichen Volumen Wasser zu vermischen und hierauf einmal mit Chloroform zu schüttelv. wodurch harzige Nebenprodukte entfernt werden. Konzentriert man dann die wasserige Lðsung wieder zum Sirup, so erhält man weit mehr und beảeutend reinere Krystalle, als wenn man der Vorschrift von $M$ arsh und $G$ a $\mathrm{r}$ d ner folgt.

Charakteristisch für die Pyrocamphonsäure ist das von den genannten Autoren nicht erwähnte $Z$ i nkalz. Man erhält es durch Stehenlassen oder Erhitzen der Säure mit viel Wasser und kohlensaurem Zink. Filtration und Verdampfen der Lösung in sehr hübschen, glänzenden Nadeln, welche in heilsem Wasser schwerer löslich sind, als in kaltem. Das trockene Salz versprüht leicht beim Erhitzen.

$0,22 \mathrm{~g}$ lufttrockenes Salz lieferten bei vorsichtigem Glühen $0,0578 \mathrm{~g}$ $\mathrm{Zn} \mathrm{O}$. entsprechend 26,27 Proz. $\mathrm{Zn}$; berechnet für $\mathrm{C}_{9} \mathrm{H}_{12} \mathrm{O}_{\mathbf{4}} \mathrm{Z}_{\mathbf{n}}$ 26,15 Proz. 
jedoch leicht verständlich, als ein besonderer Versuch uns lehrte, dals die Säure $\mathrm{C}_{9} \mathrm{H}_{14} \mathrm{O}_{4}$ durch Permanganat in der Hitze selbst leicht angegriffen wird, also unter den früher eingehaltenen Bedingungen iminer nur zufälig in minimaler Menge bestehen bleiben konnte. Deshalb wurde jetzt ein anderes Oxydationsverfahren (Vermeidung höherer Temperatur, aber Anwendung stärkerer Konzentration) benützt, dabei jedoch überraschender Weise wieder eine neve, nur einbasische Säure erhalten.

In den Mutterlaugen, welche sich nach dem Ausfällen und Abfiltrieren der Digitsäure ergaben, neutralisierten wir diesmal nur die uberschüssige Salzsäure durch Kalilauge (bis zum Verschwinden der Tropäolinreaktion) und verdampften auf dem Wasserbade, bis die Krystallisation von Chlorkalium begann. Die organischen Säuren schieden sich hierbei gröfstenteils ab und ballten sich zu einem Klumpen zusammen. Dieser wurde nach dem Erkalten fein zerrieben, mit Wasser gewaschen und getrocknet.

$\mathrm{Zu}$ einer kalten Lösung von $12 \mathrm{~g}$ dieser Masse in $360 \mathrm{~g}$ Kalilauge (1:10) wurden nun innerhalb 2-3 Tagen $16 \mathrm{~g}$ Kaliumpermanganat (in Lösung $1: 30$ ) allmählich zugegeben; die rote Farbo blieb dann ca. 3 Stunden unverändert. Nach Entfärbung durch wenig Aikohol und Filtration wurde die Lösung mit 20 Proz. ihres Gewichtes an 93 proz. Alkohol und luberschüssiger Salz.äure versetzt, worauf sich nochmals etwas Digitsäure abschied, dann neuer dings mit Kalilauge vollständig neutralisiert und ziemlich weit ein gedampft. Die konzentrierte Flüssigkeit gab jetzt mit Salzsäure einen weifsen, flockigen Niederschlag, der zum Unterschiede von der Digitsäure auf Zusatz von viel Wasser wieder verschwand und sich durch Aothr.r ausschütteln liels.

Beim Verdunsten des Aethers bleibt ein gelber, nicht krystallisierbarer Syrup. Neutralisiert man diesen mit Kalilauge und giebt zur märsig konzentrierten Lösung Chlorcalcium, so entsteht nach kurzer Zeit eine hübsche Krystallisation (derbe Nadeln odor Prismen) des $\mathrm{Kalksalzes}$ der nouen Säure, welche wir vorläufig Digsäure nennen wollen. Das Salz ist in heifsem Wasser schwerer löslich als in kaltem, kann also nicht direkt umkrystallisiert werden. Man zersetzt es deshalb zur weiteren Reinigung durch Salzsäure, zieht 
die Säure wieder durch Aether aus, neutralisiert sie und fällt abermals durch Chlorcalcium.

Das reine Salz entspricht der Formel $\left(\mathrm{C}_{8} \mathrm{H}_{11} \mathrm{O}_{3}\right)_{2} \mathrm{Ca}+6 \mathrm{H}_{8} \mathrm{O}$, die letzten Anteile des Krystallwassers entwoichen sehr schwer; die wassertreie Verbindung ist äufserst hygroskopisch.

$0,1995 \mathrm{~g}$ lufttrockenes Salz verloren bei $120^{0} 0,04 i 1 \mathrm{~g} \mathrm{H}_{2} \mathrm{O}$.

$0,1518 \mathrm{~g}$ wasserfreies Salz gaben $0,0234 \mathrm{~g} \mathrm{CaO}$.

$\begin{array}{lc}\text { Ber. für }\left(\mathrm{C}_{8} \mathrm{H}_{11} \mathrm{O}_{3}\right)_{2} \mathrm{Ca}+6 \mathrm{H}_{2} \mathrm{O} & \text { Gefunden } \\ \mathrm{H}_{2} \mathrm{O} & 23,58 \\ \text { Ber. für }\left(\mathrm{C}_{8} \mathrm{H}_{11} \mathrm{O}_{3}\right)_{2} \mathrm{Ca} & 23,60 \\ \mathrm{Ca} \quad 11,43 & \text { Gefunden } \\ \mathrm{Ca} & 11,00\end{array}$

Auch das Bary um a l z krystallisiert sehr gut, ist aber noch leichter löslich.

Schliefslich wurde aus dem ganz reinen Kalksalze die freie Digsäure durch Zersetzung mit Salzsäure und Ausschütteln mit Aether als amorphes, im Vacuum leicht anstrocknendes, sehr elektrisches Pulver gewonnen. Dasselbe löst sich leicht in Alkohol, schwer in Wasser. Aus der alkoholischen. mit Wasser gesättigten Lösung scheidet sich die Säure sehr langsam in Form von strukturlosen Körnern ab, welche gegen $130^{\circ}$ erweichen.

$0,2638 \mathrm{~g}$ vacuumtrockene Säure lieferten $0,5936 \mathrm{~g} \mathrm{CO}_{2}$ u. $0,1946 \mathrm{~g} \mathrm{H}_{2} \mathrm{O}$. Ber. für $\mathrm{C}_{8} \mathrm{H}_{12} \mathrm{O}_{3}$

C 61.54 Gefunden

$\mathrm{H} \quad \overline{1.69}$

61,37

8,19

Da eine Säure von dieser Zusammensetzung und den beschriebenen Eigenschaften unseres Wissens bisher nicht bekannt ist, war die Auffindung derselben für unseren Hauptzweck - die Aufklärung der Konstitution des Digitogenins - natürich belanglos und haben wir daraufhin auch die Versuche, auf dem bisherbeschrittenen Wege das gewünschte Ziel zu erreichen, definitiv abgebrochen.

Einwirkung von Salzsäu: e a f Digitsäure.

Auf Grund der Annahme, dafs die Digitsäure einen hydrierten Benzolkern enthält, war die Säure früher mit freiem Halogen behandelt worden, um dann durch Abspaltung von Halogenwasserstoff zu einem weniger hydrierten, also vielleicht bekannten Benzolderivat zu gelangen. Allein bei gewöhnlicher Temperatur wirken die Halogene viel zu träge auf die Säure und in der Hitze veranlafst die entstehende Halogenwasserstoffsäure reichliche Verharzung. Aussichts- 
voller erschien deshalb der umgekehrte Weg, nämlich zuerst starke Salzsäure bei niedriger Temperatur einwirken zu lassen, um wenn möglich, dadurch Wasser abzuspalten, und dann das Produkt eventuell mit freiem Halogen zu behandeln. Die in dieser Richtung angestellten Versuche ergaben thatsächlich bemerkenswerte Resultate.

$10 \mathrm{Th}$. Eisessig werden unter Eiskühlung mit trockenem Salzsäuregas gesättigt, dann fügt man $1 \mathrm{Th}$. bei $105^{0}$ getrocknete Digitsäure hinzu und leitet noch einige Zeit Salzstaure ein. Nach kurzer Zeit ist die Digitsäure gelöst unter Gelbfärbung. Man lärst die verschlossene Flasche noch 5 bis 6 Stunden in Eis stehen und gielst alsdann in ca. $300 \mathrm{Th}$. kaltes Wasser. Der entstehende, schwach gelblich gefürbte, flockige Niederschlag wird nach kurzer Zeit körnig und leicht zerreiblich. Man wäscht ihn mit kaltem Wasser und trocknet auf Thon. Das Filtrat versetzt man mit Kalilauge bis zum Verschwinden der Tropäolinreaktion und verdampft es mittelst Wasserbad bis auf $1 / 3$ seines Volumens. Dabei scheidet sich noch eine ziemliche Quantitat von Nadeln ab, welche aus derselben Substanz bestehen, wie das direkt durch Wasser gefällte Material.

Zur Reinigung wird $1 \mathrm{Th}$. des Rohproduktes in 6-8 Th. Aceton gelöst und die filtrierte Flüssigkeit mit dem gleichen Gewichte Wasser versetzt. Nach kurzer Zeit entsteht ein dicker Brei von feinen Nadeln. Diese bilden nach dem Waschen mit 50 prozentigem Alkohol und Trocknen im Vakuum eine äufserst voluminöse, filzige Masse. Sie sind chlorfrei, besitzen den Charakter einer Säure und schmelzen unter Zersetzung gegen 2450. Die Ausbeute beträgt 60 Proz.

- Uebergielst man die neue Säure mit wenig englischer Schwefelsäure, so löst sie sich mit prachtvoll roter Farbe, die aber rasch in schmutziggrün bezw. grüngelb umschlägt. Die schöne Farbenreaktion, welche die Digitsäure beim Stehonlassen mit konz. Salpetersäure zeigt, giebt die neue Säure nicht mehr.

Die Analyse ergab folgende Werte:

I. $0,1586 \mathrm{~g}$ vakuumtrockene Substanz lieferten $0,384 \mathrm{~g} \mathrm{CO}_{2}$ und $0,1138 \mathrm{~g} \mathrm{H}_{8} \mathrm{O}$.

II. $0,1388 \mathrm{~g}$ gaben $0,3366 \mathrm{~g} \mathrm{CO}_{2}$ und $0,0962 \mathrm{~g} \mathrm{H}_{2} \mathrm{O}$.

Berechnet für: $\mathrm{C}_{10} \mathrm{H}_{14} \mathrm{O}_{3}$

Gefunden:

I.

II.

$\begin{array}{rrrr}\mathrm{C} & 65,93 & 66,03 & 66,14 \\ \mathrm{H} & 7,69 & 7,97 & 7,70\end{array}$


Demnach ist aus der Digitsäure, $\mathrm{C}_{10} \mathrm{H}_{16} \mathrm{O}_{4}$, ein Mol. Wasser abgespalten worden; wir bezeichnen das Produkt zum Unterschiede von einer später noch zu beschreibenden isomeren Verbindung als a - Anhydrodigitsäure. Offenbar enthält die Digitsäure ein Hydroxyl, welches unter dem Einflusse der Eisessigchlorwasserstoffsäure entweder direkt mit einem Wasserstoffatom sich zu Wasser vereinigt oder vielleicht auch zuerst durch Chlor ersetzt wird, worauf natürlich Chlorwasserstoff sich abspalten würde. In beiden Fällen mufs eine doppelte Bindung entstehen, welche sich in der Digitsäure nicht vorfindet, und dies wird bestätigt durch die Beobachtung, dafs die $\alpha$-Anhydrodigitsäure im Gegensatze zur Digitsäure schon bei gewöhnlicher Temperatur sehr leicht von Kaliumpermanganat angegriffen wird. Merkwürdigerweise findet sich dann unter den Oxydationsprodukten eine ziemlich gro「se Menge von Digitsäure, die also auf diese Weise aus der Anhydrosäure regeneriert wird.

Die Alkalisalzlösungen der $\alpha$-Anhydrodigitsăure geben mit Chlorcalcium einen amorphen, in Alkohol löslichen Niederschlag. Vermischt man die Kalisalzlösung zuerst mit Alkohol oder Aceton und dann mit Chlorcalcium, so bilden sich langsam schöne Krystalle, die aber schon beim Versuche, sie im Vakuum zu trocknen, unter schwacher Gelbfärbung eine Zersetzung erleiden, Diese Beobachtung wurde nicht weiter verfolgt.

Die a-Anhydrodigitsäure addiert Brom und giebt bei der Behandlung mit Jodwasserstoffgas in Eisessiglösung ein krystallisierbares, jodhaltiges Derivat. Dieses liefert beim Erhitzen mit Anilin eine stickstoff haltige Verbindung, deren Lösung in Essigsäure beim Stehen an der Luft einen schönen violettroten Farbstoff absetzt. Auch diese Vorgänge bedürfen noch der weiteren Aufklärung.

Wir trachteten zunächst, aus der a-Anhydrodigitsäure noch ein weiteres Sauerstoffatom in Form von Wasser abzutrennen und studierten deshalb die

Einwirkung von Salzsăure und Essigsäureanhydrid a uf a-Anhydrodigitsäure.

Je $1 \mathrm{~g}$ Săure wird mit $4 \mathrm{~g}$ Eisessig und $4 \mathrm{~g}$ Essigsäureanhydrid ubergossen und trockenes Salzsäuregas unter Eiskühlung bis zur Sättigung eingeleitet. Allmählich tritt Lösung ein unter Rotbraun= färbung. Nach 6 stündigem Stehen der Mischung in verschlossener 
Flasche giefst man sie in Wasser, filtrirt den gelblichen Niederschlag und trocknet ihn auf Thon. Auch dieses Produkt ist eine Säure und äufserst leicht löslich in Alkohol, Aceton und Eisessig, schwer löslich in Wasser und in Aether. Löst man die Sxure in heifsem verdünntem Alkohol, so fällt sie beim Erkalten ölig aus.

Die Reinigung des Rohproduktes wird am besten in folgender Weise bewerkstelligt:

Mit einer ganz kleinen Probe der Substanz bereitet man sich eine völlig gesättigte Lösung derselben in Eisessig; diese erstarrt in 1-2 Tagen zu einem schwach violettrot gefärbten Krystallbrei.

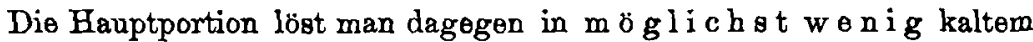
50 prozentigem Alkohol, woraus sich dann ohne aulsere Anregung auch bei sehr langem Stehen keine Krystalle absetzen. Sobald aber die aus Eisessig erzielten Kryställchen zugegeben werden, beginnt auch im verdünnten Alkohol die Ausscheidung und nach ca. 23 Stunden ist ein so dicker Brei entstanden, dafs man erst nach Verdünung mit dem halben Volumen 40 prozentigen Alkohols, weiterem eintägigem Stehenlassen und schliefslichem Zusatze des gleichen Volumens 30 prozentigen Alkohols filtrieren kann. Die so gewonnene Säure wird nochmals aus 50 prozentigem Alkohol, den man eventuell langsam verdunsten lälst, umkrystallisiert. Sio scheidet sich in krystallwasserhaltigen Nadeln oder Prismen ab, welche bei $140^{\circ} \mathrm{zu}$ erweichen beginnen, aber erst bei $170^{\circ}$ wirklich schmelzen unter Gelbfärbung. Gegen Schwefelsäure und Salpetersäure verhălt sie sich wie a-Anhydrodigitsăure.

Die weitere Untersuchung zeigte, dafs unsere Hoffnung, zu einer Verbindung von der Formel $\mathrm{C}_{10} \mathrm{H}_{12} \mathrm{O}_{2}$ zu gelangen, sich nicht erfüllt hatte. Das neue Produkt muls vielmehr auf Grund der Analyse, sowie wegen seines Verhaltens zu Alkalien als das A cet ylderiv a t einer mit der Muttersubstanz isomeren Säure, der $\beta$ - A n b y drodigitsäu re betrachtet werden.

$0,1816 \mathrm{~g}$ lufttrockene Substanz verloren bei $105^{\circ} 0,135 \mathrm{~g} \mathrm{H}_{2} \mathrm{O}$.

Berechnet fur $\mathrm{C}_{10} \mathrm{H}_{13} \mathrm{O}_{3}\left(\mathrm{C}_{2} \mathrm{H}_{3} \mathrm{O}\right)+\mathrm{H}_{2} \mathrm{O}$ Gefunden:

$$
\mathrm{H}_{2} \mathrm{O} \quad 7,43 \quad \pi, 43
$$

$0,1478 \mathrm{~g}$ wasserfreie Substanz gaben $\left.{ }^{1}\right) 0,3480 \mathrm{~g} \mathrm{CO}_{2}$ und $0,0988 \mathrm{~g} \mathrm{H}_{2} \mathrm{O}$ werden.

1) Die Verbrennung mufs sehr langsam und vorsichtig ausgeführt 
Berechnet für $\mathrm{C}_{20} \mathrm{H}_{13} \mathrm{O}_{3}\left(\mathrm{C}_{2} \mathrm{H}_{3} \mathrm{O}\right)$

$\mathrm{C} \quad 64,28$

$\mathrm{H} \quad 7,14$
Gefunden:

64,21

7,42

Durch $1 / 2$ stündiges Kochen mit überschüssiger $1 / 10$ Normalkalilauge (unter Zusatz von etwas Alkohol, um das Schäumen zu verhindern) wird die Substanz zerlegt in Essigsäure und eine neue, mit der $\alpha$-Säure isomere Verbindung, die $\beta$-Anhydrodigitsäure. Die Essigsäure wurde durch Destillation und Ueberführung in das Silbersalz (gef. 64,45 Proz. Ag ber. 64,66 Proz.) charakterisiert, das andere Spaltungsprodukt scheidet sich beim Ansäuern der alkalischen Lösung als amorpher Niederschlag aus. Dieser wird gewaschen, getrocknet und mit möglichst wenig Eisessig aufgelöst, aus dem sich bald eine körnige Krystallmasse absetzt, während harzige Nebenprodukte gelöst bleiben. Um filtrieren zu können, verdünnt man mit wenig 50 prozentiger Essigsäure; das so gereinigte und wieder getrocknete Material wird in Aceton gelöst, dio Lösung in einen Kolben filtriert und der freiwilligen Verdunstung überlassen, wobei die Säure in langen, strahlenförmig anschielsenden, seidenglänzenden Nadeln effloresciert. Die Säure wird durch Einwirkung von Licht. und Luft leicht gelb; sie schmilzt unter Zersetzung bei 262-2630. Zur weiteren Untersuchung derselben fehlte uns bisher leider das nötige Material.

Wie entsteht nun aber die Acetyl- $\beta$-Anhydrodigitsäure aus der $\alpha$-Säure?

Die Digitsäure enthält nach den früher mitgeteilten Beobachtungen kein Aldehyd- oder Keton-Radikal; die zwei Sauerstoffatome, welche sich in ihr aulserhalb des Carboxyls noch vorfinden, können also nur entweder Hydroxylgruppen angehören oder zur Verkettung von je 2 Kohlenstoffatomen dienen, d. h. als sog. "Brückensauerstoff“ vorliegen, wio dies z: B. beim Cineol angenommen wird. Die erstere Art der Bindung (Hydroxylform) gilt nun höchst wabrscheinlich für den Sauerstoff, welcher bei der Bildung der $a$-Anhydrodigitsäure eliminiert wird; die zweite dagegen dürfte nach allen vorliegenden Beobachtungen zutreffen für das zweite Sauerstoffatom und sie veranlafst vermutlich die Entstehung der Acetyl- $\beta$-Anhydrodigitsäure. Nimmt man nämlich an, dals ein solcher "Brickensauerstoff" vorhanden ist, sowie dafs die a-Anhydrodigitsäure einen teilweise hydrirten 
Benzolkern enthält, und bedenkt man endlich, dafs Essigsäureanhydrid und trockene Chlorwasserstoffsäure leicht Acetylchlorid liefern können, so lälst sich die Bildung der Acetyl - $\beta$-Anhydrodigitsäure in folgender Weise erklären:

I.<smiles>CCC1CCCC(C)C1CC</smiles>

$$
+\mathrm{C}_{2} \mathrm{H}_{3} \mathrm{OCl}=
$$<smiles>CCOC1(C)C2CC3C(Cl)C2CC1C3(C)Cl</smiles>

II.
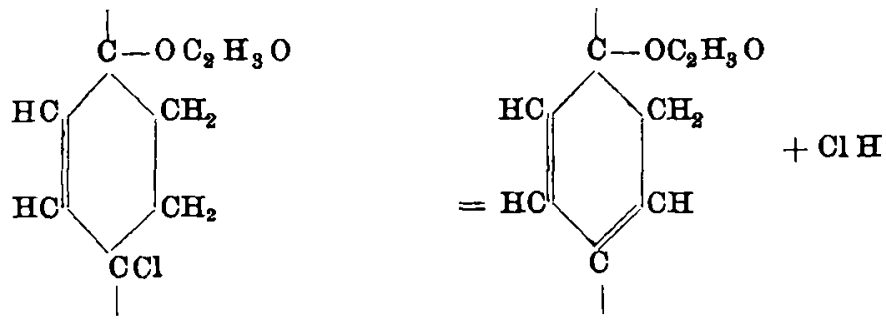

Natürlich sollte dann die Säure auch einfach mittelst Acetylchlorid erhältlich sein, was noch zu probieren ist. Erweist sich nun diese Auffassung bei weiteren Versuchen als richtig, so würde die Auffindung obiger Reaktion einen ganz wesentlichen Fortschritt in der Aufklärung der Konstitution des Digitogenins und seiner Derivate bedeuten. Denn gerade durch die Beseitigung der eigenartigen Bindung des einen Sauerstoffatoms wäre jedenfalls die Wahrscheinlichkeit, dafs man jetzt zu relativ einfachen Verbindungen von bekannter Konstitution gelangen kann, bedeutend näher gerückt, und die $\beta$-Anhydrodigitsäure mülste ja nach obigen Erörterungen einfach eine hydroxylierte Dihydrocuminsäure sein.

Leider ist im Augenblicke unser Material völlig verbraucht und neues in der nötigen Menge zur Zeit überhaupt nicht za erhalten. Sobald wir aber wieder Digitonin besitzen, soll die Untersuchung fortgesetzt werden.

München, im Juni 1894. 